\title{
NOTE ON THE PROBLEM OF MOTION OF VISCOUS FLUID AROUND A ROTATING AND TRANSLATING RIGID BODY
}

\author{
Paul Deuring $^{a}$, Stanislav KraČmaR $^{b, c}$, Šárka NeČasová ${ }^{c, *}$ \\ ${ }^{a}$ Université du Littoral Côte d'Opale, Centre Universitaire de la Mi-Voix 50, rue F.Buisson CS 80699, 62228 \\ Calais Cedex, France \\ ${ }^{b}$ Czech Technical University in Prague, Faculty of Mechanical Engineering, Department of Technical \\ Mathematics, Karlovo nám. 13, 12135 Praha 2, Czech Republic \\ ${ }^{c}$ Czech Academy of Sciences, Institute of Mathematics, Žitná 25, 11567 Praha 1, Czech Republic \\ * corresponding author: matus@math.cas.cz
}

Abstract. We consider the linearized and nonlinear systems describing the motion of incompressible flow around a rotating and translating rigid body $\mathcal{D}$ in the exterior domain $\Omega=\mathbb{R}^{3} \backslash \overline{\mathcal{D}}$, where $\mathcal{D} \subset \mathbb{R}^{3}$ is open and bounded, with Lipschitz boundary. We derive the $L^{\infty}$-estimates for the pressure and investigate the leading term for the velocity and its gradient. Moreover, we show that the velocity essentially behaves near the infinity as a constant times the first column of the fundamental solution of the Oseen system. Finally, we consider the Oseen problem in a bounded domain $\Omega_{R}:=B_{R} \cap \Omega$ under certain artificial boundary conditions on the truncating boundary $\partial B_{R}$, and then we compare this solution with the solution in the exterior domain $\Omega$ to get the truncation error estimate.

KEYWORDS: Incompressible fluid, rigid body, exterior domain, estimates of pressure, leading terms, artificial boundary conditions.

\section{INTRODUCTION}

The boundary problem of Navier-Stokes equations describing flows past a rigid body translating with a constant velocity (with or without rotation) is one of the challenging problems in fluid mechanics. In recent decades, much effort has been made to analyze the properties of solutions of both stationary and non-stationary solutions, both linear and nonlinear mathematical models, both in the whole space and in exterior domains. The difficulty which arises in this type of problem is the variability of the spatial domain in time. To solve it there are two possibilities: (i) to study a problem in the time dependent domain, see Conca, Starovoitov and Tucsnak [1], Desjardins and Esteban [2], Gunzburger, Lee and Seregin [3], Hoffman and Starovoitov [4, etc. (ii) to use a transformation in order to transform the spatial domain varying in time in to a fixed domain. For this approach the global or local transformation can be applied. The global linear transformation implies that the whole space is rigidly rotated and shifted back to its original position at each time $t>0$ (cf. [5]). The equations of motion of the fluid-rigid body system is in a frame attached to the rigid body, with its origin in the center of mass of the latter and coinciding with an inertial frame at time $t=0$. (Works related to this type of transformation see [6-12]). The local transformation implies that the change of variables only acts in a bounded neighbourhood of the body, the solenoidal condition of the fluid velocity are preserved and the regularity of the solution are not changed. See e.g. works of Tucsnak, Cumsille and Takahashi (cf. [13-15]).

\subsection{Formulation of the PROBlem}

Let us formulate our problem in the fixed domain, which is a result of applying the global linear transformation, for more details, see [5]. The systems of equations are as follows

$$
\begin{aligned}
-\Delta u(z)+\tau \partial_{1} u(z)- & (\omega \times z) \cdot \nabla u(z)+\omega \times u(z) \\
& +\tau(u(z) \cdot \nabla) u(z)+\nabla \pi(z)=F(z) \\
& \operatorname{div} u(z)=0 \text { for } z \in \Omega \\
-\Delta u(z)+\tau \partial_{1} u(z)- & (\omega \times z) \cdot \nabla u(z)+\omega \times u(z)+\nabla \pi(z)=F(z) \\
& \operatorname{div} u(z)=0 \text { for } z \in \Omega
\end{aligned}
$$

where $\mathcal{D} \subset \mathbb{R}^{3}$ is open and bounded, with Lipschitz boundary. The systems $\sqrt{1.1}$ and $[1.2$ together with some boundary conditions on $\partial \Omega=\partial \mathcal{D}$ constitute the mathematical models (linear and non-linear, respectively) describing the stationary flow of a viscous incompressible fluid around a rigid body which moves at a constant velocity and rotates at a constant angular velocity. In this study we consider that the rotation is parallel to the velocity at infinity. (For more details concerning the derivation of the model, see [5, 7]. The description and the 
analysis in the case where the rotation is not parallel to the velocity at infinity can be found in the following works, see [16, 17]).

The aim is to obtain the $L^{\infty}$ estimates for the pressure in the linear and nonlinear cases, since such estimates are missing in the literature. Only the estimates of the velocity field and the gradient of the velocity field in $L^{\infty}$ are available. This implies that complete information about the decay of the solution $(u, \pi)$ of the systems 1.1 , 1.2 for $|x| \rightarrow \infty$. (For other works see [18], [19].)

Second, we are interested in the "Leray solutions" of (1.1), supplemented by a decay condition at infinity,

$$
u(x) \rightarrow 0 \text { for }|x| \rightarrow \infty,
$$

and the suitable boundary conditions on $\partial \Omega$. Weak solutions are characterized by the conditions $u \in L^{6}(\Omega)^{3} \cap$ $W_{l o c}^{1,1}(\Omega)^{3}, \nabla u \in L^{2}(\Omega)^{9}$ and $\pi \in L_{l o c}^{2}(\Omega)$.

From [18] and [20, it follows that the velocity part of the Leray solution $(u, \pi)$ in 1.1 and $(1.3)$ decays for $|x| \rightarrow \infty$ as the estimates express below

$$
|u(x)| \leq C(|x| s(x))^{-1}, \quad|\nabla u(x)| \leq C(|x| s(x))^{-3 / 2}
$$

for $x \in \mathbb{R}^{3}$ with $|x|$ sufficiently large, where $s(x):=1+|x|-x_{1}\left(x \in \mathbb{R}^{3}\right)$ and $C>0$ a constant independent of $x$. The factor $s(x)$ may be considered as a mathematical manifestation of the wake extending downstream behind a body moving in a viscous fluid. In the work by M. Kyed, (see [21]) it was shown that

$$
u_{j}(x)=\gamma E_{j 1}(x)+R_{j}(x), \quad \partial_{l} u_{j}(x)=\gamma \partial_{l} E_{j 1}(x)+S_{j l}(x) \quad\left(x \in \overline{\mathfrak{D}}^{c}, 1 \leq j, l \leq 3\right),
$$

where $E: \mathbb{R}^{3} \backslash\{0\} \mapsto \mathbb{R}^{4} \times \mathbb{R}^{3}$ denotes a fundamental solution to the Oseen system

$$
-\Delta v+\tau \partial_{1} v+\nabla \varrho=f, \operatorname{div} v=0 \quad \text { in } \mathbb{R}^{3} .
$$

The term $E_{j 1}(x)$ can be expressed explicitly in terms of elementary functions. The coefficient $\gamma$ is also given explicitly, its definition involving the Cauchy stress tensor. The remainding terms $R$ and $S$ are characterized by the relations $R \in L^{q}(\Omega)^{3}$ for $q \in(4 / 3, \infty), S \in L^{q}(\Omega)^{3}$ for $q \in(1, \infty)$. From [22, Section VII.3] it is known that $E_{j 1} \mid B_{r}^{c} \notin L^{q}\left(B_{r}^{c}\right)$ for $r>0, q \in[1,2]$, and $\partial_{l} E_{j 1} \mid B_{r}^{c} \notin L^{q}\left(B_{r}^{c}\right)$ for $r>0, q \in[1,4 / 3], j, l \in\{1,2,3\}$. The function $R$ decays faster than $E_{j 1}$, and $S_{j l}$ decays faster than $\partial_{l} E_{j 1}$, in the sense of $L^{q}$-integrability. Thus, the equations in 1.5 can be viewed in fact as asymptotic expansions of $u$ and $\nabla u$, respectively. Let us mention that the result in 21] are valid under the assumption that $u$ verifies the boundary conditions

$$
u(x)=e_{1}+(\omega \times x) \text { for } x \in \partial \Omega,
$$

which is not our case.

Reference [21] does not deal with $L^{\infty}$-decay of $R$ and $S$, nor does it indicate whether $S=\nabla R$.

Below, in Theorem 4.1 we derive an $L^{\infty}$-decay of $u$ and $\nabla u$ respectively, which is independent on the boundary conditions. However, in comparison with [21] and indicated in $[1.5$, our leading term is less explicit than the term $\gamma E_{j 1}(x)$ in $(1.5)$ and instead of the fundamental solution $E_{j 1}(x)$ of the stationary Oseen system, we use the time integral of the fundamental solution of the evolutionary Oseen system.

In [23] it was proved that $\mathcal{Z}_{j 1}(x, 0)=E_{j 1}(x)$ for $x \in \mathbb{R}^{3} \backslash\{0\}, 1 \leq j \leq 3$, and $\lim _{|x| \rightarrow \infty}\left|\partial_{x}^{\alpha} \mathcal{Z}_{j k}(x, 0)\right|=$ $O\left((|x| s(x))^{-3 / 2-|\alpha| / 2}\right)$ for $1 \leq j \leq 3, k \in\{2,3\}$ ([23, Corollary 4.5, Theorem 5.1]). Thus, setting

$$
\mathfrak{G}_{j}(x):=\sum_{k=2}^{3} \beta_{k} \mathcal{Z}_{j k}(x, 0)+\mathfrak{F}_{j}(x) \quad\left(x \in{\overline{B_{S_{1}}}}^{c}, 1 \leq j \leq 3\right),
$$

we may obtain from 4.3 that

$$
u_{j}(x)=\beta_{1} E_{j 1}(x)+\left(\int_{\partial \Omega} u \cdot n d o_{x}\right) x_{j}\left(4 \pi|x|^{3}\right)^{-1}+\mathfrak{G}_{j}(x) \quad\left(x \in{\overline{B_{S_{1}}}}^{c}, 1 \leq j \leq 3\right)
$$

and

$$
\lim _{|x| \rightarrow \infty}\left|\partial^{\alpha} \mathfrak{G}(x)\right|=O\left((|x| s(x))^{-3 / 2-|\alpha| / 2} \ln (2+|x|)\right) \quad \text { for } \alpha \in \mathbb{N}_{0}^{3} \text { with }|\alpha| \leq 1
$$

(Theorem 4.2, Corollary 4.3).

Comparing the coefficient $\gamma$ from $(1.5)$ in the work [21] with the coefficient $\beta_{1}$ from $(1.9)$ in [24], see Theorem 4.1 below, and taking into account the boundary condition (1.7) in [21, it follows that $\gamma$ and $\beta_{1}$ are equal.

Third, we are solving the linear system 1.2 in a truncation $\Omega_{R}:=B_{R} \cap \Omega$ of the exterior domain $\mathbb{R}^{3} \backslash \overline{\mathcal{D}}$ under certain artificial boundary conditions on the truncating boundary $\partial B_{R}$. Then we compare this solution with the solution of 1.2 in the exterior domain, i.e. to find the error estimates of the method of an artificial boundary condition. For this aim we use $L^{\infty}$-estimates of the velocity and of the pressure. 


\section{Definitions And nOtATion}

Let us define

$$
s(y):=1+|y|-y_{1} \text { for } y \in \mathbb{R}^{3}, \Omega=\mathbb{R}^{3} \backslash \overline{\mathcal{D}}, \Omega_{R}:=B_{R} \cap \Omega, B_{R}^{c}:=\mathbb{R}^{3} \backslash \overline{B_{R}},
$$

where $B_{R}:=\left\{x \in \mathbb{R}^{3} ;|x|<R\right\}$, for $R>0$ such that $B_{R} \supset \overline{\mathcal{D}}$.

So, $\Omega_{R}$ is the truncation of the exterior domain $\Omega=\mathbb{R}^{3} \backslash \overline{\mathcal{D}}$ by the ball $B_{R}$. The boundary $\Omega_{R}$ consists of parts $\partial \Omega$ and $\partial B_{R}$, the later we call the truncating boundary.

Fix $\tau \in(0, \infty), e_{1}:=(1,0,0), \omega=|\omega| e_{1},|\omega| \neq 0$, and $\Omega:=|\omega|\left(\begin{array}{ccc}0 & 0 & 0 \\ 0 & 0 & -1 \\ 0 & 1 & 0\end{array}\right)$.

So, $\Omega \cdot z=\omega \times z$ for $z \in \mathbb{R}^{3}$. For $U \subset \mathbb{R}^{3}$ open, $u \in W_{\text {loc }}^{2,1}(U)^{3}, z \in U$, put

$$
\begin{aligned}
(L u)(z) & :=-\Delta u(z)+\tau \partial_{1} u(z)-(\omega \times z) \cdot \nabla u(z)+\omega \times u(z), \\
\left(L^{*} u\right)(z) & :=-\Delta u(z)-\tau \partial_{1} u(z)+(\omega \times z) \cdot \nabla u(z)-\omega \times u(z) .
\end{aligned}
$$

Put $N(x):=(4 \pi|x|)^{-1}$ for $x \in \mathbb{R}^{3} \backslash\{0\}$ ("Newton potential", fundamental solution of the Poisson equation in $\left.\mathbb{R}^{3}\right), \mathfrak{O}(x):=(4 \pi|x|)^{-1} e^{-\tau\left(|x|-x_{1}\right) / 2}$ for $x \in \mathbb{R}^{3} \backslash\{0\}$ (fundamental solution of the scalar Oseen equation $-\Delta v+\tau \partial_{1} v=g$ in $\left.\mathbb{R}^{3}\right)$,

$$
\begin{array}{ll}
\text { Put } & K(z, t):=(4 \pi t)^{-3 / 2} e^{-|z|^{2} /(4 t)} \quad\left(z \in \mathbb{R}^{3}, t \in(0, \infty)\right), \\
\Lambda(z, t):=\left(K(z, t) \delta_{j k}+\partial z_{j} \partial z_{k}\left(\int_{\mathbb{R}^{3}}(4 \pi|z-y|)^{-1} K(y, t) d y\right)\right)_{1 \leq j, k \leq 3}\left(z \in \mathbb{R}^{3}, t>0\right), \\
\Gamma(x, y, t):=\Lambda\left(x-\tau t e_{1}-e^{-t \Omega} y, t\right) \cdot e^{-t \Omega}, \\
\widetilde{\Gamma}(x, y, t):=\Lambda\left(x+\tau t e_{1}-e^{t \Omega} y, t\right) \cdot e^{t \Omega} \quad\left(x, y \in \mathbb{R}^{3}, t>0\right), \\
\mathcal{Z}(x, y):=\int_{0}^{\infty} \Gamma(x, y, t) d t, \widetilde{\mathcal{Z}}(x, y):=\int_{0}^{\infty} \widetilde{\Gamma}(x, y, t) d t, \quad\left(x, y \in \mathbb{R}^{3}, x \neq y\right) .
\end{array}
$$

$\psi(r):=\int_{0}^{r}\left(1-e^{-t}\right) t^{-1} d t \quad(r \in \mathbb{R}), \quad \Phi(x):=(4 \pi \tau)^{-1} \psi\left(\tau\left(|x|-x_{1}\right) / 2\right) \quad\left(x \in \mathbb{R}^{3}\right)$,

$E_{j k}(x):=\left(\delta_{j k} \Delta-\partial_{j} \partial_{k}\right) \Phi(x), \quad E_{4 k}(x):=x_{k}\left(4 \pi|x|^{3}\right)^{-1} \quad\left(x \in \mathbb{R}^{3} \backslash\{0\}, 1 \leq j, k \leq 3\right)$ (fundamental solution of the Oseen system 1.6 , with $\left(E_{j k}\right)_{1 \leq j, k \leq 3}$ the velocity part and $\left(E_{4 k}\right)_{1 \leq k \leq 3}$ the pressure part).

For $q \in(1,2), f \in L^{q}\left(\mathbb{R}^{3}\right)^{3}$, put

$$
\mathcal{R}(f)(x):=\int_{\mathbb{R}^{3}} \mathcal{Z}(x, y) f(y) d y \quad\left(x \in \mathbb{R}^{3}\right)
$$

see [25, Lemma 3.1].

We will use the space $D_{0}^{1,2}(\Omega)^{3}:=\left\{v \in L^{6}(\Omega)^{3} \cap H_{l o c}^{1}(\Omega)^{3}: \nabla v \in L^{2}(\Omega)^{9}, v \mid \partial \Omega=0\right\}$ equipped with the norm $\|\nabla u\|_{2}$, where $v \mid \partial \Omega$ means the trace of $v$ on $\partial \Omega$. For $p \in(1, \infty)$, define $M_{p}$ as the space of all pairs of functions $(u, \pi)$ such that $u \in W_{l o c}^{2, p}(\Omega)^{3}, \pi \in W_{l o c}^{1, p}(\Omega)$,

$$
\begin{gathered}
u\left|\Omega_{R} \in W^{1, p}\left(\Omega_{R}\right)^{3}, \quad \pi\right| \Omega_{R} \in L^{p}\left(\mathcal{D}_{R}\right), \quad u \mid \partial \Omega \in W^{2-1 / p, p}(\partial \Omega)^{3}, \\
\operatorname{div} u\left|\Omega_{R} \in W^{1, p}\left(\Omega_{R}\right), \quad L(u)+\nabla \pi\right| \Omega_{R} \in L^{p}\left(\Omega_{R}\right)^{3}
\end{gathered}
$$

for some $R \in(0, \infty)$ with $\bar{\Omega}^{c} \subset B_{R}$.

We write $C$ for generic constants. In order to romove possible ambiguities, we sometimes use the notation $C\left(\gamma_{1}, \ldots, \gamma_{n}\right)$ in order to indicate that the constant in question depends particularly on $\gamma_{1}, \ldots, \gamma_{n} \in(0, \infty)$, for some $n \in \mathbb{N}$. But the relevant constant may depend on other parameters as well.

\section{Decay estimates}

In the first part of this section, we recall some known results from [25] and [26] about the decay of the velocity part of the solution of the system 1.2 . In order to get the full decay characterization of the solution, we derive the decay of the pressure part of the solution of $(1.2)$. In the second part of this section, we extend the result for the pressure to the non-linear case of (1.1). 


\subsection{Decay estimates in the Linear CaSe}

Our starting point is a decay result from [26] for the velocity part $u$ of a solution to 1.2 .

Theorem 3.1. ([26, Theorem 3.12]) Suppose that $\Omega^{c}$ is $C^{2}$-bounded. Let $p \in(1, \infty),(u, \pi) \in M_{p}$. Put $F=L(u)+\nabla \pi$. Suppose there are numbers $S_{1}, S, \gamma \in(0, \infty), A \in[2, \infty), B \in \mathbb{R}$ such that $S_{1}<S$,

$$
\begin{gathered}
\Omega^{c} \cup \operatorname{supp}(\operatorname{div} u) \subset B_{S_{1}}, \quad u\left|B_{S}^{c} \in L^{6}\left(B_{S}^{c}\right)^{3}, \quad \nabla u\right| B_{S}^{c} \in L^{2}\left(B_{S}^{c}\right)^{9}, \\
A+\min \{1, B\} \geq 3,|F(z)| \leq \gamma|z|^{-A} s(z)^{-B} \text { for } z \in B_{S_{1}}^{c} .
\end{gathered}
$$

Then

$$
\begin{gathered}
|u(y)| \leq C(|y| s(y))^{-1} l_{A, B}(y), \\
|\nabla u(y)| \leq C(|y| s(y))^{-3 / 2} s(y)^{\max (0,7 / 2-A-B)} l_{A, B}(y)
\end{gathered}
$$

for $y \in B_{S}^{c}$, where function $l_{A, B}$ is given by

$$
\left\{\begin{array}{cc}
1 & \text { if } \quad A+\min \{1, B\}>3 \\
\max (1, \ln (y)) & \text { if } \quad A+\min \{1, B\}=3
\end{array}\right.
$$

Corollary 3.2. Let $p \in(1, \infty), \gamma, S_{1}, S \in(0, \infty)$ with $\Omega^{c} \subset B_{S_{1}}, S_{1}<S, A \in[2, \infty), B \in \mathbb{R}$ with $A+\min \{1, B\} \geq 3$. Let $F: \Omega \mapsto \mathbb{R}^{3}$ be measurable with $F \mid \Omega_{S_{1}} \in L^{p}\left(\Omega_{S_{1}}\right)^{3}$ and $|F(z)| \leq \gamma|z|^{-A} s(z)^{-B}$ for $z \in$ $B_{S_{1}}^{c}$.

Let $u \in W_{l o c}^{1, p}(\Omega)^{3}$ with $u\left|B_{S}^{c} \in L^{6}\left(B_{S}^{c}\right)^{3}, \nabla u\right| B_{S}^{c} \in L^{2}\left(B_{S}^{c}\right)^{9}, \operatorname{supp}(\operatorname{div} u) \subset B_{S_{1}}$,

$$
\begin{aligned}
& \int_{\overline{\mathcal{D}}^{c}}\left[\nabla u \cdot \nabla \varphi+\left(\tau \partial_{1} u-(\omega \times z) \cdot \nabla u+(\omega \times u)-F\right) \cdot \varphi\right] d z \\
& =0 \text { for } \varphi \in C_{0}^{\infty}(\Omega)^{3} \text { with } \operatorname{div} \varphi=0 .
\end{aligned}
$$

Then inequalities (3.1) and (3.2) hold for $y \in B_{S}^{c}$.

Moreover $F \in L^{q}(\Omega)^{3}$ for $q \in(1, p]$. If $p \geq 6 / 5$, the function $F$ may be considered as a bounded linear functional on $\mathcal{D}_{0}^{1,2}(\Omega)^{3}$, in the usual sense.

Let $\pi \in L_{l o c}^{p}(\Omega)$ with

$$
\begin{gathered}
\int_{\overline{\mathcal{D}}^{c}}\left[\nabla u \cdot \nabla \varphi+\left(\tau \partial_{1} u-(\omega \times z) \cdot \nabla u+(\omega \times u)-F\right) \cdot \varphi\right. \\
-\pi \operatorname{div} \varphi] d z=0 \quad \text { for } \varphi \in C_{0}^{\infty}(\Omega)^{3} .
\end{gathered}
$$

Fix some number $S_{0} \in\left(0, S_{1}\right)$ with $\overline{\mathcal{D}} \cup \operatorname{supp}(\operatorname{div} u) \subset B_{S_{0}}$. Then the relations $u \mid{\overline{B_{S_{0}}}}^{c} \in W_{l o c}^{2, p}\left({\overline{B_{S_{0}}}}^{c}\right)^{3}, \pi \in$ $W_{\text {loc }}^{1, p}\left({\overline{B_{S_{0}}}}^{c}\right)$ and $L\left(u \mid{\overline{B_{S_{0}}}}^{c}\right)+\nabla \pi=F \mid{\overline{B_{S_{0}}}}^{c}$ hold.

The main result of this section, dealing with the $L^{\infty}$-estimates of the pressure, is stated in

Theorem 3.3. Let $p, \gamma, S_{1}, S, A, B, F, u$ be given as in Corollary 3.2, but with the stronger assumptions $A=5 / 2, B \in(1 / 2, \infty)$ on $A$ and $B$. Let $\pi \in L_{\text {loc }}^{p}(\Omega)$ such that 3.4 holds Then there is $c_{0} \in \mathbb{R}$ such that

$$
\left|\pi(x)+c_{0}\right| \leq C|x|^{-2} \quad \text { for } x \in B_{S}^{c} .
$$

Corollary 3.4. Let $p, \gamma, S_{1}, S, A, B, F, u$ be given as in Corollary 3.2 but with the stronger assumptions $A \geq 5 / 2, A+\min \{1, B\}>3$ on $A$ and $B$. Let $\pi \in L_{l o c}^{p}(\Omega)$ such that (3.4) holds. Then there is $c_{0} \in \mathbb{R}$ such that inequality (3.5) is valid.

Proof: Put $B^{\prime}:=A-5 / 2+\min \{1, B\}$. Since $A+\min \{1, B\}>3$, we have $B^{\prime} \in(1 / 2, \infty)$. Moreover, since $A \geq 5 / 2$, we find for $z \in B_{S_{1}}^{c}$ that

$$
|F(z)| \leq \gamma C\left(S_{1}, A\right)|z|^{-5 / 2} s(z)^{-A+5 / 2-B} \leq \gamma C\left(S_{1}, A\right)|z|^{-5 / 2} s(z)^{-B^{\prime}} .
$$

Thus the assumptions of Theorem 3.3 are satisfied with $B$ replaced by $B^{\prime}$ and with a modified parameter $\gamma$. This implies the conclusion of Theorem 3.3 


\subsection{DeCAy estimates in the NON-Linear CASE}

Let us assume now the non-linear case, i.e. the system 1.1. First, recall the result about the decay properties of the velocity in this non-linear case:

Theorem 3.5. [20, Theorem 1.1] Let $\gamma, S_{1} \in(0, \infty), p_{0} \in(1, \infty), A \in(2, \infty), B \in[0,3 / 2]$ with $\Omega^{c} \subset B_{S_{1}}$, $A+\min \{B, 1\}>3, A+B \geq 7 / 2$. Take $F: \mathbb{R}^{3} \mapsto \mathbb{R}^{3}$ measurable with $F \mid B_{S_{1}} \in L^{p_{0}}\left(B_{S_{1}}\right)^{3}$,

$$
|F(y)| \leq \gamma \cdot|y|^{-A} \cdot s(y)^{-B} \text { for } y \in B_{S_{1}}^{c} .
$$

Let $u \in L^{6}(\Omega)^{3} \cap W_{l o c}^{1,1}(\Omega)^{3}, \pi \in L_{l o c}^{2}(\Omega)$ with $\nabla u \in L^{2}(\Omega)^{9}$, div $u=0$ and

$$
\begin{aligned}
& \int_{\overline{\mathcal{D}}^{c}}\left[\nabla u \cdot \nabla \varphi+\tau \partial_{1} u-(\omega \times z) \cdot \nabla u+\omega \times u\right. \\
&+\tau(u \cdot \nabla) u-F) \cdot \varphi-\pi \operatorname{div} \varphi] \mathrm{d} x=0
\end{aligned}
$$

for $\varphi \in C_{0}^{\infty}(\Omega)^{3}$. Let $S \in\left(S_{1}, \infty\right)$. Then

$$
\left|\partial^{\alpha} u(x)\right| \leq C(|x| s(x))^{-1-|\alpha| / 2} \text { for } x \in B_{S}^{c}, \alpha \in \mathbb{N}_{0}^{3} \text { with }|\alpha| \leq 1 .
$$

Now, using Theorems 3.3 and 3.5 we are in the position to prove the result on the decay of the pressure in the non-linear case:

Theorem 3.6. Consider the situation in Theorem 3.5. Suppose in addition that $A \geq 5 / 2$. Then there is $c_{0} \in \mathbb{R}$ such that inequality (3.5) holds.

\section{LEADING TERM}

In this section we study the asymptotic behavior of the velocity profile of the system $(1.2)$. Let us recall known results from [26] and [24].

Theorem 4.1. Let $\mathcal{D} \subset \mathbb{R}^{3}$ be open, $p \in(1, \infty), f \in L^{p}\left(\mathbb{R}^{3}\right)^{3}$ with supp $(f)$ compact. Let $S_{1} \in(0, \infty)$ with $\overline{\mathcal{D}} \cup \operatorname{supp}(f) \subset B_{S_{1}}, \Omega=\overline{\mathcal{D}}^{c}$.

Let $u \in L^{6}(\Omega)^{3} \cap W_{l o c}^{1,1}(\Omega)^{3}, \pi \in L_{\text {loc }}^{2}(\Omega)$ with $\nabla u \in L^{2}(\Omega)^{9}$, div $u=0$ and

$$
\begin{aligned}
& \int_{\Omega}\left[\nabla u \cdot \nabla \varphi+\left(\tau \partial_{1} u+\tau(u \cdot \nabla) u-(\omega \times z) \cdot \nabla u+\omega \times u\right) \cdot \varphi-\pi \operatorname{div} \varphi\right] d z \\
& =\int_{\Omega} f \cdot \varphi d z \text { for } \varphi \in C_{0}^{\infty}(\Omega)^{3} .
\end{aligned}
$$

(This means the pair $(u, \pi)$ is a Leray solution to 1.2 , (1.3).) Suppose in addition that

$$
\Omega^{c} \text { is } C^{2} \text {-bounded, } \quad u\left|\partial \Omega \in W^{2-1 / p, p}(\partial \Omega)^{3}, \quad \pi\right| B_{S_{1}} \backslash \overline{\mathcal{D}} \in L^{p}\left(B_{S_{1}} \backslash \overline{\mathcal{D}}\right) .
$$

Let $n$ denote the outward unit normal to $\Omega$, and define

$$
\begin{aligned}
& \beta_{k}:=\int_{\Omega} f_{k}(y) d y \\
& +\int_{\partial \Omega} \sum_{l=1}^{3}\left(-\partial_{l} u_{k}(y)+\delta_{k l} \pi(y)+\left(\tau e_{1}-\omega \times y\right)_{l} u_{k}(y)-\tau\left(u_{l} u_{k}\right)(y)\right) n_{l}(y) d o_{y}
\end{aligned}
$$

for $1 \leq k \leq 3$,

$$
\begin{aligned}
& \mathfrak{F}_{j}(x):=\int_{\Omega}\left[\sum_{k=1}^{3}\left(\mathcal{Z}_{j k}(x, y)-\mathcal{Z}_{j k}(x, 0)\right) f_{k}(y)-\tau \cdot \sum_{k, l=1}^{3} \mathcal{Z}_{j k}(x, y)\left(u_{l} \partial_{l} u_{k}\right)(y)\right] d y \\
& +\int_{\partial \Omega} \sum_{k=1}^{3}\left[\left(\mathcal{Z}_{j k}(x, y)-\mathcal{Z}_{j k}(x, 0)\right) \sum_{l=1}^{3}\left(-\partial_{l} u_{k}(y)+\delta_{k l} \pi(y)+\left(\tau e_{1}-\omega \times y\right)_{l} u_{k}(y)\right) n_{l}(y)\right. \\
& \quad+\left(E_{4 j}(x-y)-E_{4 j}(x)\right) u_{k}(y) n_{k}(y) \\
& \left.\quad+\sum_{l=1}^{3}\left(\partial y_{l} \mathcal{Z}_{j k}(x, y)\left(u_{k} n_{l}\right)(y)+\tau \mathcal{Z}_{j k}(x, 0)\left(u_{l} u_{k} n_{l}\right)(y)\right)\right] d o_{y}
\end{aligned}
$$


for $x \in{\overline{B_{S_{1}}}}^{c}, 1 \leq j \leq 3$. The preceding integrals are absolutely convergent. Moreover $\mathfrak{F} \in C^{1}\left({\overline{B_{S_{1}}}}^{c}\right)^{3}$ and equation

$$
u_{j}(x)=\sum_{k=1}^{3} \beta_{k} \mathcal{Z}_{j k}(x, 0)+\left(\int_{\partial \Omega} u \cdot n d o_{x}\right) x_{j}\left(4 \pi|x|^{3}\right)^{-1}+\mathfrak{F}_{j}(x) .
$$

holds. In addition, for any $S \in\left(S_{1}, \infty\right)$, there is a constant $C>0$ which depends on $\tau, \omega, S_{1}, S, f, u$ and $\pi$, and which is such that

$$
\left|\partial^{\alpha} \mathfrak{F}(x)\right| \leq C(|x| s(x))^{-3 / 2-|\alpha| / 2} \ln (2+|x|) \quad \text { for } x \in{\overline{B_{S}}}^{c}, \alpha \in \mathbb{N}_{0}^{3} \text { with }|\alpha| \leq 1
$$

Theorem 4.2. Let $\mathfrak{D}, p, f, S_{1}, u, \pi$ satisfy the assumptions of Theorem 4.1, including (4.2). Let $\beta_{1}, \beta_{2}, \beta_{3}$ and $\mathfrak{F}$ be defined as in Theorem 4.1. Define the function $\mathfrak{G}$ as

$$
\mathfrak{G}_{j}(x):=\sum_{k=2}^{3} \beta_{k} \mathcal{Z}_{j k}(x, 0)+\mathfrak{F}_{j}(x) \quad\left(x \in{\overline{B_{S_{1}}}}^{c}, 1 \leq j \leq 3\right)
$$

Then $\mathfrak{G} \in C^{1}\left(\overline{B_{S_{1}}}\right)^{3}$, equation

$$
u_{j}(x)=\beta_{1} E_{j 1}(x)+\left(\int_{\partial \Omega} u \cdot n d o_{x}\right) x_{j}\left(4 \pi|x|^{3}\right)^{-1}+\mathfrak{G}_{j}(x) \quad\left(x \in{\overline{B_{S_{1}}}}^{c}, 1 \leq j \leq 3\right)
$$

holds, and for any $S \in\left(S_{1}, \infty\right)$, there is a constant $C>0$ which depends on $\tau, \omega, S_{1}, S, f, u$ and $\pi$, and which is such that

$$
\left|\partial^{\alpha} \mathfrak{G}(x)\right| \leq C(|x| s(x))^{-3 / 2-|\alpha| / 2} \ln (2+|x|) \quad \text { for } x \in{\overline{B_{S}}}^{c}, \alpha \in \mathbb{N}_{0}^{3} \text { with }|\alpha| \leq 1
$$

Corollary 4.3. Take $\mathcal{D}, p, f, S_{1}, u, \pi$ as in Theorem 4.1, but without requiring (4.2). (This means that $(u, \pi)$ is only assumed to be a Leray solution of 1.2 , 1.3). $)$ Put $\widetilde{p}:=\min \{3 / 2, p\}$. Then $u \in W_{\text {loc }}^{2, \tilde{p}}(\Omega)^{3}$ and $\pi \in W_{\text {loc }}^{1, \tilde{p}}(\Omega)$.

Fix some number $S_{0} \in\left(0, S_{1}\right)$ with $\overline{\mathcal{D}} \cup \operatorname{supp}(f) \subset B_{S_{0}}$, and define $\beta_{1}, \beta_{2}, \beta_{3}$ and $\mathfrak{F}$ as in Theorem 4.1 . but with $\mathcal{D}$ replaced by $B_{S_{0}}$, and $n(x)$ by $S_{0}^{-1} x$, for $x \in \partial B_{S_{0}}$. Moreover, define $\mathfrak{G}$ as in $(4.5)$. Then all the conclusions of Theorem 4.2 are valid.

\section{Formulation of THE PROBlEM With ARTIFICIAL BOUNDARY CONDitions}

Recall that we defined $\Omega_{R}=B_{R} \cap \Omega$. We introduce the subspace $W_{R}$ of $H^{1}\left(\Omega_{R}\right)$ denoting

$$
W_{R}:=\left\{v \in H^{1}\left(\Omega_{R}\right)^{3}: v \mid \partial \Omega=0\right\}
$$

where $v \mid \partial \Omega$ means the trace of $v$ on $\partial \Omega$.

Lemma 5.1. ([27, Lemma 4.1]) The estimate

$$
\|u\|_{2} \leq C\left(R\|\nabla u\|_{2}+R^{1 / 2}\left\|u \mid \partial B_{R}\right\|_{2}\right)
$$

holds for $R \in(0, \infty)$ with $\Omega^{c} \subset B_{R}$ and for $u \in W_{R}$.

We introduce an inner product $(\cdot, \cdot)^{(R)}$ in $W_{R}$ by defining

$$
(v, w)^{(R)}=\int_{\Omega_{R}} \nabla v \cdot \nabla w d x+\int_{\partial B_{R}}(\tau / 2) v \cdot w d o_{x} \text { for } v, w \in W_{R} .
$$

The space $W_{R}$ equipped with this inner product is a Hilbert space. The norm generated by this scalar product $(\cdot, \cdot)^{(R)}$ is denoted by $|\cdot|^{(R)}$, that is

$$
|v|^{(R)}:=\left(\|\nabla v\|_{2}^{2}+(\tau / 2)\left\|v \mid \partial B_{R}\right\|_{2}^{2}\right)^{1 / 2} \text { for } v \in W_{R}
$$


We define the bilinear forms

$$
\begin{aligned}
& \mathcal{A}_{R}: H^{1}\left(\Omega_{R}\right)^{3} \times H^{1}\left(\Omega_{R}\right)^{3} \rightarrow \mathbb{R}, \\
& \mathcal{B}_{R}: H^{1}\left(\Omega_{R}\right)^{3} \times L^{2}\left(\Omega_{R}\right) \rightarrow \mathbb{R}, \\
& \mathcal{A}_{R}(u, w):= \int_{\Omega_{R}}\left[\nabla u \cdot \nabla w+\tau \partial_{1} u \cdot w\right] d x+\frac{\tau}{2} \int_{\partial B_{R}}(u(x) \cdot w(x))\left(1-\frac{x_{1}}{R}\right) d o_{x}, \\
& \int_{\Omega_{R}}[-((\omega \times x) \cdot \nabla) u(x)+(\omega \times u(x))] \cdot w(x) d x \\
& \mathcal{B}_{R}(w, \sigma):=\left.-\int_{\Omega_{R}}(\operatorname{div} w) \sigma d x,+(\omega \times u(x))\right] \cdot w(x) d x \\
& \text { for } u, w \in H^{1}\left(\Omega_{R}\right)^{3}, \sigma \in L^{2}\left(\Omega_{R}\right), R \in(0, \infty) \text { with } \Omega^{c} \subset B_{R} .
\end{aligned}
$$

Lemma 5.2. Let $R \in(0, \infty)$ with $\Omega^{c} \subset B_{R}$. Then

$$
\left|\mathcal{A}_{R}(u, w)\right| \leq C(R)|u|^{(R)}|w|^{(R)}
$$

for $u, w \in H^{1}\left(\Omega_{R}\right)^{3}$.

The key observation in this section is stated in the following lemma, which is the basis of the theory presented in this section.

Lemma 5.3. Let $R \in(0, \infty)$ with $\Omega^{c} \subset B_{R}$, and let $w \in W_{R}$. Then the equation $\left(|w|^{(R)}\right)^{2}=\mathcal{A}_{R}(w, w)$ holds.

Proof: Using the definition $\mathcal{A}_{R}(\cdot, \cdot)$, we get

$$
\begin{aligned}
\mathcal{A}_{R}(w, w)= & \int_{\mathcal{D}_{R}}\left[|\nabla w|^{2}+\tau \partial_{1}\left(\frac{|w|^{2}}{2}\right)-(\omega \times x) \cdot \nabla\left(\frac{|w|^{2}}{2}\right)\right] d x \\
& +\frac{\tau}{2} \int_{\partial B_{R}}|w(x)|^{2}\left(1-\frac{x_{1}}{R}\right) d o_{x} \\
= & \int_{\mathcal{D}_{R}}|\nabla w|^{2} d x+\int_{\partial B_{R}}\left(\frac{\tau}{2}|w(x)|^{2} \frac{x_{1}}{R}-\frac{1}{2}(\omega \times x) \cdot \frac{x}{R}|w(x)|^{2}\right) d o_{x} \\
& +\frac{\tau}{2} \int_{\partial B_{R}}|w(x)|^{2}\left(1-\frac{x_{1}}{R}\right) d o_{x} \\
= & \int_{\mathcal{D}_{R}}|\nabla w|^{2} d x+\frac{\tau}{2} \int_{\partial B_{R}}|w(x)|^{2}=\left(|w|^{(R)}\right)^{2}
\end{aligned}
$$

We applied that $(\omega \times x) \cdot x=0$ for $x, \omega \in \mathbb{R}^{3}$.

As in [28], we obtain that the bilinear form $\beta_{R}$ is stable:

Theorem 5.4. ([28, Corollary 4.3]) Let $R>0$ with $\Omega^{c} \subset B_{R}$. Then

$$
\inf _{\rho \in L^{2}\left(\Omega_{R}\right), \rho \neq 0} \sup _{v \in W_{R}, v \neq 0} \frac{\mathcal{B}_{R}(v, \rho)}{|v|^{(R)}\|\rho\|_{2}} \geq C(R) .
$$

We note that functions from $W_{l o c}^{1,1}(\Omega)$ with $L^{2}$-integrable gradient are $L^{2}$-integrable on truncated exterior domains:

Lemma 5.5. [29, Lemma II.6.1] Let $w \in W_{l o c}^{1,1}(\Omega)$ with $\nabla w \in L^{2}(\Omega)^{3}$, and let $R \in(0, \infty)$ with $\Omega^{c} \subset B_{R}$. Then $w \mid \Omega_{R} \in L^{2}\left(\Omega_{R}\right)$. In particular the trace of $w$ on $\partial \Omega$ is well defined.

The preceding lemma is implicitly used in the ensuing theorem, where we introduce an extension operator $\mathfrak{E}: H^{1 / 2}(\partial \Omega)^{3} \mapsto W_{l o c}^{1,1}(\Omega)^{3}$ such that $\operatorname{div} \mathfrak{E}(b)=0$.

Theorem 5.6. [29, Exercise III.3.8] There is an operator $\mathfrak{E}$ from $H^{1 / 2}(\partial \Omega)^{3}$ into $W_{\text {loc }}^{1,1}(\Omega)^{3}$ satisfying the relations $\nabla \mathfrak{E}(b) \in L^{2}(\Omega)^{9}, \mathfrak{E}(b) \mid \partial \Omega=b$ and $\operatorname{div} \mathfrak{E}(b)=0$ for $b \in H^{1 / 2}(\partial \Omega)^{3}$.

In view of Lemma 5.2 and 5.3 and Theorem 5.6 and 5.4 , the theory of mixed variational problems yields 
Theorem 5.7. Let $S>0$ with $\Omega^{c} \subset B_{S}, R \in[2 S, \infty), F \in L^{6 / 5}\left(\Omega_{R}\right)^{3}, b \in H^{1 / 2}(\partial \Omega)^{3}$. Then there is a uniquely determined pair of functions $(\widetilde{V}, P)=(\widetilde{V}(R, F, b), P(R, F, b)) \in W_{R} \times L^{2}\left(\Omega_{R}\right)$ such that

$$
\begin{aligned}
& \mathcal{A}_{R}(\widetilde{V}, g)+\mathcal{B}_{R}(g, P)=\int_{\mathcal{D}_{R}} F \cdot g d x-\mathcal{A}_{R}\left(\mathfrak{E}(b) \mid \Omega_{R}, g\right) \text { for } g \in W_{R}, \\
& \mathcal{B}_{R}(\widetilde{V}, \sigma)=0 \text { for } \sigma \in L^{2}\left(\Omega_{R}\right),
\end{aligned}
$$

where the operator $\mathfrak{E}$ was introduced in Theorem 5.6 .

Let us interpret variational problem (5.1), (5.2) as a boundary value problem. Define the expression used in the boundary condition on the artificial boundary $\partial B_{R}$ :

$$
\mathcal{L}_{R}(u, \pi)(x):=\left(\sum_{j=1}^{3} \partial_{j} u_{k}(x) \frac{x_{j}}{R}-\pi(x) \frac{x_{k}}{R}+\frac{\tau}{2}\left(1-\frac{x_{1}}{R}\right) u_{k}(x)\right) 1 \leq k \leq 3
$$

for $x \in \partial B_{R}, R \in(0, \infty)$ with $\overline{\mathcal{D}} \subset B_{R}, u \in W^{2,6 / 5}\left(\Omega_{R}\right)^{3}, \pi \in W^{1,6 / 5}\left(\Omega_{R}\right)$.

Lemma 5.8. Assume that $\Omega^{c}$ is $\mathcal{C}^{2}$-bounded. Let $S \in(0, \infty)$ with $\Omega^{c} \subset B_{S}, R \in[2 S, \infty), F \in L^{6 / 5}\left(\Omega_{R}\right)^{3}$ and $b \in W^{7 / 6,6 / 5}(\partial \Omega)^{3}$. Put $V:=\tilde{V}(R, F, b)+\mathfrak{E}(b) \mid \Omega_{R}$, with $V(R, F, b)$ from Theorem 5.7 and $\mathfrak{E}(b)$ from Theorem 5.6. Suppose that $V \in W^{2,6 / 5}\left(\Omega_{R}\right)^{3}$ and $P=P(R, F, b) \in W^{1,6 / 5}\left(\Omega_{R}\right)$, with $P(R, F, b)$ also introduced in Theorem 5.7. Then

$$
\begin{gathered}
-\Delta V(z)+\tau \partial_{1} V(z)-(\omega \times z) \cdot \nabla V(z)+\omega \times V(z)+\nabla P(z)=F(z) \\
\operatorname{div} V(z)=0
\end{gathered}
$$

for $z \in \Omega_{R}$, and $V \mid \partial \Omega=b, \quad \mathcal{L}_{R}(V, P)=0$.

Theorem 5.9. Suppose that $\Omega^{c}$ is $C^{2}$-bounded. Let $\gamma, S_{1} \in(0, \infty)$ with $\Omega^{c} \subset B_{S_{1}}, A \in[5 / 2, \infty), B \in \mathbb{R}$ with $A+\min \{1, B\}>3$. Let $F: \Omega \mapsto \mathbb{R}^{3}$ be measurable with $F \mid \Omega_{S_{1}} \in L^{6 / 5}\left(\Omega_{S_{1}}\right)^{3}$ and $|F(z)| \leq \gamma|z|^{-A} s(z)^{-B}$ for $z \in$ $B_{S_{1}}^{c}$.

Let $b \in W^{7 / 6,6 / 5}(\partial \Omega)^{3}, u \in W_{l o c}^{1,1}(\Omega)^{3} \cap L^{6}(\Omega)^{3}$ such that $\nabla u \in L^{2}(\Omega)^{9}$, div $u=0, u \mid \partial \Omega=b$ and equation (3.3) is satisfied.

For $R \in\left[2 S_{1}, \infty\right)$, put $V_{R}:=\widetilde{V}(R, F, b)+\mathfrak{E}(b)$, with $\mathfrak{E}(b)$ from Theorem 5.6 , and $\widetilde{V}(R, F, b)$ from Theorem 5.7. Then

$$
|u|_{\Omega_{R}}-\left.V_{R}\right|^{(R)} \leq C R^{-1} \text { for } R \in[2 S, \infty)
$$

\section{ACKNOWLEDGEMENTS}

The works of S.K. and Š. N. were supported by Grant No. 19-04243S of GAČR in the framework of RVO 67985840, S.K. is supported by RVO 12000 .

\section{REFERENCES}

[1] C. Conca, J. San Martín H., M. Tucsnak. Motion of a rigid body in a viscous fluid. Comptes Rendus de l'Académie des Sciences - Series I - Mathematics 328(6):473 - 478, 1999. DOI:10.1016/S0764-4442(99)80193-1

[2] B. Desjardins, M. J. Esteban. Existence of weak solutions for the motion of rigid bodies in a viscous fluid. Archive for Rational Mechanics and Analysis 146:59-71, 1999. DOI:10.1007/s002050050136

[3] M. Gunzburger, H.-C. Lee, G. Seregin. Global existence of weak solutions for viscous incompressible flows around a moving rigid body in three dimensions. Journal of Mathematical Fluid Mechanics 2:219-266, 2000. DOI:10.1007/PL00000954.

[4] K. H. Hoffmann, V. N. Starovoitov. On a motion of a solid body in a viscous fluid. Two dimensional case. Advances in Mathematical Sciences and Applications 9:633-648, 1999.

[5] G. P. Galdi. Handbook of Mathematical Fluid Dynamics, vol. 1, chap. On the motion of a rigid body in a viscous liquid: A mathematical analysis with applications, pp. 653-791. North-Holland, Amsterdam, 2002.

[6] R. Farwig, M. Krbec, Š. Nečasová. A weighted $L^{q}$-approach to Oseen flow around a rotating body. Mathematical Methods in the Applied Sciences 31(5):551-574, 2008. DOI:10.1002/mma.925

[7] R. Farwig. An $l^{q}$-analysis of viscous fluid flow past a rotating obstacle. Tôhoku Math J 58:129-147, 2006. DOI: $10.2748 / \mathrm{tmj} / 1145390210$

[8] R. Farwig. Estimates of lower order derivatives of viscous fluid flow past a rotating obstacle. Regularity and other aspects of the Navier-Stokes equations 70:73-84, 2005. 
[9] R. Farwig, T. Hishida, D. Muller. Lq-theory of a singular "winding" integral operator arising from fluid dynamics. Pacific Journal of Mathematics 215:297-313, 2004. DOI:10.2140/pjm.2004.215.297.

[10] T. Hishida. An existence theorem for the Navier-Stokes flow in the exterior of a rotating obstacle. Archive for Rational Mechanics and Analysis 150:307-348, 1999. DOI:10.1007/s002050050190

[11] T. Hishida. The Stokes operator with rotation effect in exterior domains. Analysis 19(1):51 - 68, 1999. DOI:10.1524/anly.1999.19.1.51

[12] T. Hishida. $l^{q}$ estimates of weak solutions to the stationary Stokes equations around a rotating body. Journal of the Mathematical Society of Japan 58:743-767, 2006.

[13] T. Takahashi. Analysis of strong solutions for the equations modeling the motion of a rigid-fluid system in a bounded domain. Advances in Differential Equations 8(12):1499 - 1532, 2003.

[14] T. Takahashi, T. Marius. Global strong solutions for the two-dimensional motion of an infinite cylinder in a viscous fluid. Journal of Mathematical Fluid Mechanics 6:53-77, 2004. DOI:10.1007/s00021-003-0083-4.

[15] P. Cumsille, T. Takahashi. Well posedness for the system modelling the motion of a rigid body of arbitrary form in an incompressible viscous fluid. Czechoslovak Mathematical Journal 58:961-992, 2008. DOI:10.1007/s10587-008-0063-2

[16] R. Farwig, R. Guenther, E. Thomann, Š. Nečasová. The fundamental solution of linearized nonstationary Navier-Stokes equations of motion around a rotating and translating body. Discrete and Continuous Dynamical Systems 34:511-529, 2014. DOI:10.3934/dcds.2014.34.511

[17] M. Geissert, T. Hansel. A non-autonomous model problem for the Oseen-Navier-Stokes flow with rotating effects. Journal of The Mathematical Society of Japan 63, 2010. DOI:10.2969/jmsj/06331027

[18] G. P. Galdi, M. Kyed. Steady-state Navier-Stokes flows past a rotating body: Leray solutions are physically reasonable. Archive for Rational Mechanics and Analysis 200:21-58, 2011. DOI:10.1007/s00205-010-0350-6

[19] R. Farwig, G. Galdi, M. Kyed. Asymptotic structure of a leray solution to the navier-stokes flow around a rotating body. Pacific Journal of Mathematics 253(2):367-382, 2011. DOI:10.2140/pjm.2011.253.367

[20] P. Deuring, S. Kračmar, Š. Nečasová. Pointwise decay of stationary rotational viscous incompressible flows with nonzero velocity at infinity. Journal of Differential Equations 255(7):1576 - 1606, 2013. DOI:10.1016/j.jde.2013.05.016

[21] M. Kyed. On the asymptotic structure of a Navier-Stokes flow past a rotating body. Journal of the Mathematical Society of Japan 66(1):1-16, 2014. DOI:10.2969/jmsj/06610001

[22] G. P. Galdi. An Introduction to the Mathematical Theory of the Navier-Stokes Equations, vol. I. Linearised Steady Problems. Springer, New York, 1998.

[23] P. Deuring, S. Kračmar, Š. Nečasová. Asymptotic structure of viscous incompressible flow around a rotating body, with nonvanishing flow field at infinity. Zeitschrift für angewandte Mathematik und Physik 68, 2016. DOI:10.1007/s00033-016-0760-x

[24] P. Deuring, S. Kračmar, Š. Nečasová. A leading term for the velocity of stationary viscous incompressible flow around a rigid body performing a rotation and a translation. Discrete and Continuous Dynamical Systems 37:1389-1409, 2016. DOI:10.3934/dcds.2017057.

[25] P. Deuring, S. Kračmar, Š. Nečasová. On pointwise decay of linearized stationary incompressible viscous flow around rotating and translating bodies. SIAM J Math Analysis 43:705-738, 2011. DOI:10.1137/100786198

[26] P. Deuring, S. Kračmar, Š. Nečasová. Linearized stationary incompressible flow around rotating and translating bodies - leray solutions. Discrete and Continuous Dynamical Systems - Series S 7:967-979, 2014. DOI:10.3934/dcdss.2014.7.967.

[27] P. Deuring. Finite element methods for the stokes system in three-dimensional exterior domains. Mathematical Methods in the Applied Sciences 20(3):245-269, 1997. DOI:10.1002/(SICI)1099-1476(199702)20:3<245::AID-MMA856>3.0.CO;2-F

[28] P. Deuring, S. Kračmar. Artificial boundary conditions for the oseen system in 3D exterior domains. Analysis 20(1):65 - 90, 2000. DOI:10.1524/anly.2000.20.1.65

[29] G. P. Galdi. An introduction to the mathematical theory of the Navier-Stokes equations. Springer, New York, 2nd edn., 2011. 